\title{
A discrete network model to represent the deformation behavior of human amnion
}

\author{
Arabella Mauri ${ }^{\mathrm{a}, 1}$, Raoul Hopf ${ }^{\mathrm{a}}$, Alexander E. Ehret ${ }^{\mathrm{a}, \mathrm{c}}$, Catalin R. Picu ${ }^{\mathrm{b}}$, \\ Edoardo Mazza ${ }^{\mathrm{a}, \mathrm{c}}$ \\ ${ }^{a}$ Department of Mechanical and Process Engineering, ETH Zurich, 8092 Zurich, \\ Switzerland \\ ${ }^{b}$ Department of Mechanical, Aerospace and Nuclear Engineering, Rensselaer Polytechnic \\ Institute, Troy, NY 12180, USA \\ ${ }^{c}$ Empa. Swiss Federal Laboratories for Materials Science and Technology, 8600 \\ Dübendorf, Switzerland
}

\begin{abstract}
A discrete network model (DNM) to represent the mechanical behavior of the human amnion is proposed. The amnion is modeled as randomly distributed points interconnected with connector elements representing collagen crosslinks and fiber segments, respectively. This DNM is computationally efficient and allows simulations with large domains. A representative set of parameters has been selected to reproduce the uniaxial tension-stretch and kinematic responses of the amnion. Good agreement is found between the predicted and measured equibiaxial tension-stretch curves. Although the model represents the amnion phenomenologically, model parameters are physically motivated and their effect on the tension-stretch and in-plane kinematic responses is discussed. The model is used to investigate the local response in the near field of a circular hole, revealing that the kinematic response at the circular free boundaries leads to compaction and strong alignment of the
\end{abstract}

Email address: mauri@imes.mavt.ethz.ch ()

Preprint submitted to J Mech Behav Biomed Mater

October 16, 2015

(C) 2015. This manuscript version is made available under the Elsevier user license http://www.elsevier.com/open-access/userlicense/1.0/ 
network at the border of the defect.

Keywords: Human amnion, Discrete Network Model, Microstructural

Alignment, Nonaffinity

\section{Introduction}

The mechanical behavior of the human amnion resembles the one of a textile in that there is a strong coupling between elongation in loading direction and in-plane deformation in uniaxial tensile loading, leading to very large transverse contraction (Bürzle and Mazza, 2013; Lake and Barocas, 2011), high biaxial stiffness and high toughness (Bürzle et al., 2013; Oyen et al., 2005; Perrini et al., 2015). The fibrous nature of the amnion emerges directly from observation of its microstructure (see Fig. 1), mainly consisting of cross-linked in-plane distributed collagen fibers (with no preferred orientation), and including few cells in the trophoblast layer and the epithelial monolayer (Hollenstein, 2011; Mauri et al., 2013). The non-fibrillar matrix largely consists of water, which is easily expelled from the collagenous network during deformation (Mauri et al., 2015a). Thus, the microstructure of the human amnion motivates the choice of a discrete network model (DNM) to represent its mechanical behavior (cf. Chandran and Barocas, 2006; Koh and Oyen, 2012b). DNMs have been developed to investigate the mechanical behavior of fibrous materials such as e.g. paper (e.g. Isaksson and Hägglund, 2009; Kulachenko and Uesaka, 2012) and electrospun networks (e.g. Koh and Oyen, 2015; Carleton et al., 2015). Discrete approaches have also been adopted to represent biological fibrous materials, such as the actin network in the cytoskeleton (e.g. Huisman et al., 2007; Pritchard et al., 2014), the 


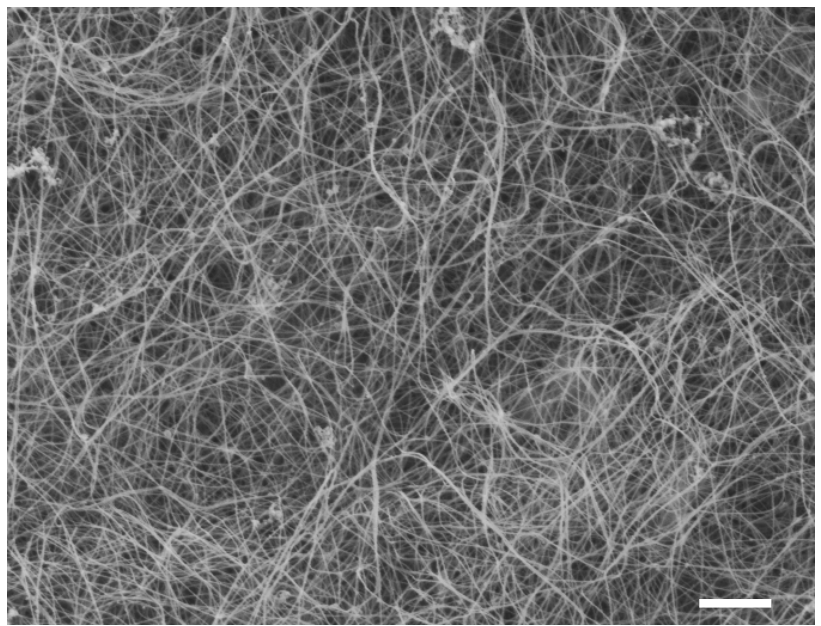

Figure 1: Top view of the collagenous network in the amnion with scanning electron microscopy, showing no preferred orientation (cf. Hollenstein (2011)), courtesy of M. Hollenstein. Scale bar: $2 \mu \mathrm{m}$.

arterial wall (e.g. Stylianopoulos and Barocas, 2007a; Shah et al., 2014), collagen type I matrices (e.g. Abhilash et al., 2014; Wang et al., 2014), and collagenous gel tissue equivalents (e.g. Chandran and Barocas, 2006; Hadi and Barocas, 2013). These fibrous materials are usually modeled as random athermal and semiflexible networks, i.e. with long filaments not subject to thermal fluctuation and for which the energy is stored in bending and axial modes (Picu, 2011; Pritchard et al., 2014; Broedersz and MacKintosh, 2014). DNMs are computationally expensive due to the representation of each single filament with either a Timoshenko or Euler-Bernoulli beam. For this reason, the macroscopic response of these DNMs is usually computed by use of a representative volume element (RVE) or a representative area element (RAE). In the past years, much effort has been invested in understanding the nonaffine deformation behavior of these random semiflexible networks (cf. Picu, 
2011). Two distinct types of behavior have been identified (Shahsavari and Picu, 2012). In the non-affine deformation range, most of the deformation energy is stored in the bending mode and is characterized by strong size effects, related to the large (strain and energy) heterogeneity (Shahsavari and Picu, 2013). The affine deformation is observed in networks with higher density and/or higher bending to axial stiffness ratio. In this range most of the energy is stored in the axial and shear deformation modes of the fibers. Koh and Oyen (2012a,b) developed a DNM to investigate toughening mechanisms activated at the notch tip of fibrous membranes and electrospun scaffolds. Partial bonding between fibers (i.e. sparse network) was found to enhance network flexibility around the defect, which enables a large microstructural rearrangement around the notch and increases the toughness of the material. These results are consistent with the experimental observations reported in Koh et al. (2013). The introduction of nonlinear fiber properties (elastic, plastic and damage regimes) allowed to reproduce the ductile and brittle behavior of electrospun networks by considering large failure strains, progressive damage of the fibers and brittle fibers with small failure strains in the model (Koh and Oyen, 2015). Onck et al. (2005) developed a two-dimensional network, later extended to three dimensions (Huisman et al., 2007; Zagar et al., 2015), to investigate the nature of the nonlinear strain-stiffening response. These authors found that nonlinearity arises from the transition between the bending- and stretching-dominated regimens irrespectively of the undulation of the fibers and the properties of the crosslinks. A network model based on the experimentally characterized fiber geometry was proposed by D'Amore et al. (2014) and the macroscopic response was validated with respect to ex- 
perimental results for different types of electrospun networks. Stylianopoulos and Barocas $(2007 \mathrm{a}, \mathrm{b})$ proposed a multiscale approach based on volume averaging. An RVE was defined by a DNM to capture the non-affine response of the collagen network and coupled at the macroscopic scale with an isotropic neo-Hookean matrix material. This model was able to capture the response of collagenous gels (Stylianopoulos and Barocas, 2007b; Chandran and Barocas, 2007; Hadi and Barocas, 2013) and arterial walls (Stylianopoulos and Barocas, 2007a; Shah et al., 2014), where the stress contributions of matrix and collagen network were additively combined like springs in a parallel arrangement. A coupled model was successively developed by Zhang et al. (2013b,a), where the nonfibrillar matrix was included in the RVE. By this structural coupling, the matrix constrains the collagenous network towards a more affine deformation, generating heterogeneous stress and strain fields, thus indicating the possible relevance of representing the specific fiber-matrix interaction of the considered tissue.

In this study, we propose a DNM for the amnion based on a computationally efficient framework, which allows simulating a very large representative area or the entire specimen. The model can be applied to investigate clinically relevant configurations, for which the effect of local heterogeneous deformation is important. After a sensitivity study, a specific parameter set was chosen, which is able to represent the monotonic uniaxial and biaxial response of the amnion. The results of the DNM were used to rationalize the experimentally observed behavior of amnion. Moreover, the change in microstructure simulated by the model was analyzed with image analysis tools similarly to that used for multiphoton microscopy (MPM) studies and the results were 
comparatively discussed.

(a)

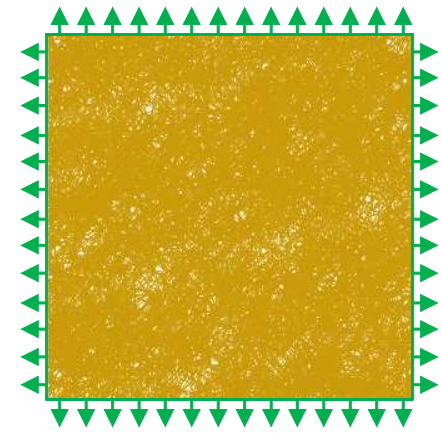

(d)

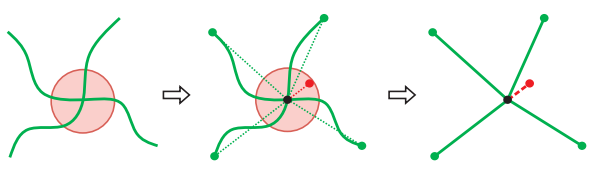

(b)

(c)
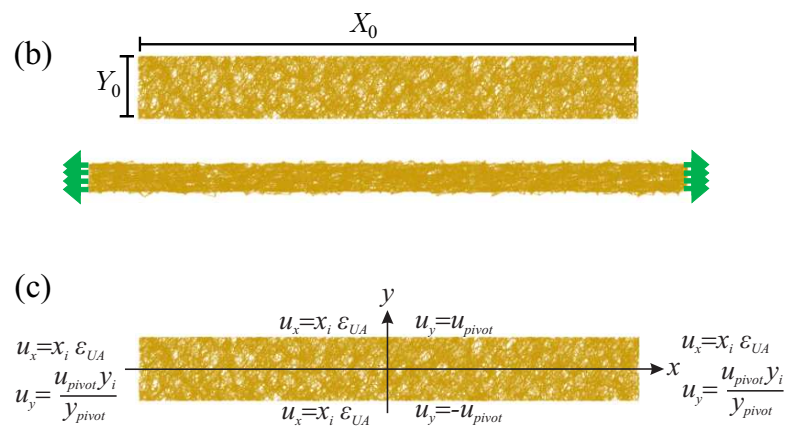

(e)

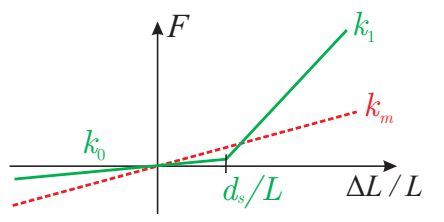

Figure 2: Models used for (a) biaxial and (b) uniaxial loading of the network. (c) boundary conditions for the uniaxial case. (d) schematic representation of the procedure used to generate the network. (e) force-strain curves representing the behavior of the two types of connectors.

\section{Methods}

\subsection{Geometry definition}

The amniotic microstructure is represented by a sparse network, i.e. crosslinks are not placed at all fiber crossing. This central force fiber network is generated from the idealization of two crosslinked fibers and an additional short-range interaction, see Fig. 2d. Thus, altogether each crosslink has coordination number of 5, i.e. five connections to other crosslinks. The geometry is generated in a $2 \mathrm{D}$ domain of dimensions $\left(X_{0}, Y_{0}\right)$ using a custom Matlab (R2013a, The MathWorks, Inc.) program which takes the following steps: 
1. $N_{0}$ crosslinks are placed at randomly selected locations in the domain, which is divided in square cells of edge length $L_{c}$, where $L_{c} \ll X_{0}$.

2. Four connecting fibers are defined for each crosslink (green continuous lines in Fig. 2d). The four connecting points are selected randomly from the eight neighboring cells of the central crosslink. This leads to a distribution of connector lengths with a mean value $\approx L_{c}$.

3. The fifth connecting fiber (red dashed line in Fig. 2d) is introduced to ensure physical stability and its constitutive behavior is different from that of the other fibers, as described in Sec. 2.2. This fiber links the representative crosslink with a randomly selected node located within a circle of radius $L_{c}$. Its length is therefore less than $L_{c}$.

4. The boundary region of the domain is trimmed out to ensure that the network geometry is statistically uniform across the problem domain. The dangling fibers resulting from this operation are connected with a set of nodes defined along this newly defined boundary.

Geometric percolation, i.e. the connection of every crosslink to the network, is ensured from the connecting algorithm and is checked after the geometry is generated. Stiffness percolation, i.e. non-zero modulus at small strains, is ensured by the fact that crosslinks have a coordination number of five. In planar pin-joined central force networks the smallest coordination number required for stability is 4 (Maxwell, 1864). In our case, due to the stochastic nature of the algorithm to generate the four connections, it is possible that towards the end of the crosslinking process, there are not enough free neighbors in an appropriate distance to be connected, so that the average coordination number drops slightly below four and an additional connection 
is needed. Note that the mean connectivity of the network is significantly higher than 4 (but slightly below 5) and this insures global stability.

\subsection{Mechanical behavior of connectors}

Fiber segments are modeled using axial connector elements (CONN3D2) in Abaqus (Abaqus 6.10-EF1, DS Simulia Corp., Providence RI, USA), and all crosslinks are of pin-joint type. The elastic behavior is defined differently for (i) the four connectors representing the fiber segments and (ii) the fifth connector. The force-strain curve of the fiber connectors (i) is bi-linear, as shown in Fig. 2f (green continuous line). The compressive stiffness, $k_{0}$, is much smaller than the tensile stiffness $k_{1}$ (cf. Table 1) and approximates the force contribution associated with fiber bending during compression and at small tensile strains when the fiber crimp is gradually stretched out. The break point that separates the two regimes is defined at a non-zero strain $d_{s} / L$. The length $d_{s}$ is a parameter of the model and quantifies the amount of slack (crimp) of fibers in the initial, unloaded configuration. Since the initial distance between cross-links $L$ varies around a mean in the order of $L_{c}$, there is a distribution of the slack strain $d_{s} / L$ within the network such that fibers spanning long distances have an already reduced slack strain compared to those connecting neighboring points. The fifth connector (ii) is considered linear elastic and of stiffness $k_{m}$ much smaller than $k_{1}$ and might represent a soft interaction of fibers with their surrounding. These parameters were selected (Table 1) such that the predicted uniaxial response closely matched the tension-stretch and kinematic response of the amnion reported by Bürzle and Mazza (2013). Further verification of the model is performed using biaxial data as discussed below. 
A damping component, defined as an additional viscous element in parallel to the elastic component of the connectors, is introduced to stabilize the network numerically. This damping element generates a force opposite and proportional to the relative velocity of the two nodes of each connector. The damping parameter $\eta$ is chosen enough small $(\eta=0.01[\mathrm{Ns} / \mathrm{mm}])$ so that its effect on the global tension-stretch response is minimal and the network is numerically stable. Fig. 3 shows the influence of $\eta$ on the calculated nominal tension $\mathrm{T}$ for the applied strain rate of $0.75 \% / \mathrm{s}$.

\begin{tabular}{lcccccccc}
\hline & $k_{0}$ & $k_{1}$ & $d_{s}$ & $k_{m}$ & $X_{0}$ & $Y_{0}$ & $N_{0}$ & $L_{c}$ \\
& {$[\mathrm{~N}]$} & {$[\mathrm{N}]$} & {$[\mathrm{mm}]$} & {$[\mathrm{N}]$} & {$[\mathrm{mm}]$} & {$[\mathrm{mm}]$} & {$[-]$} & {$[\mathrm{mm}]$} \\
\hline Uniaxial & 0.0001 & 1.5 & 0.03 & 0.01 & 80 & 14 & 8000 & 1 \\
Equibiaxial & 0.0001 & 1.5 & 0.03 & 0.01 & 34 & 34 & 8000 & 1 \\
\hline
\end{tabular}

Table 1: Geometrical and connector characteristics for reference sets.

\subsection{Simulations of mechanical tests}

Simulations of domains representative of uniaxial (80 mm x $14 \mathrm{~mm}$ ) and equibiaxial (34 mm x $34 \mathrm{~mm}$ ) specimens are performed using the finite element solver Abaqus (implicit, static) within half hour computational time. The total deformation energy of the network is the sum of the deformation energy in all connectors and is minimized within Abaqus. The global nominal tension $\mathrm{T}[\mathrm{N} / \mathrm{mm}]$ in the elongation direction was computed as the sum of the reaction forces at the corresponding boundary, divided by its initial width $\left(Y_{0}\right)$. The macroscopic deformation behavior is imposed by boundary conditions for each boundary point as indicated in Fig. 2. For the biaxial case, the boundary points of all edges are displaced affinely according to the 
global stretch. Special boundary conditions are defined for the uniaxial configuration to ensure that free edges are free of traction (see Fig. 2c). To this end, all points of one edge are coupled (in the transverse direction) with a free pivot point so that all boundary points of the edge experience the same displacement, representing a mean value of the individual displacements.

As an example of application, the DNM is used to analyze the effect of a hole. A squared domain $\left(X_{0}, X_{0}\right)$ with a circular hole (diameter $D \approx 10 L_{c} \approx$ $\left.0.1 X_{0}\right)$ under equibiaxial extension applied to the boundaries is considered. As an effective and simple representation of the hole, the connectors within a circular area of diameter $D$ are assigned a stiffness two orders of magnitude smaller than the other connectors.
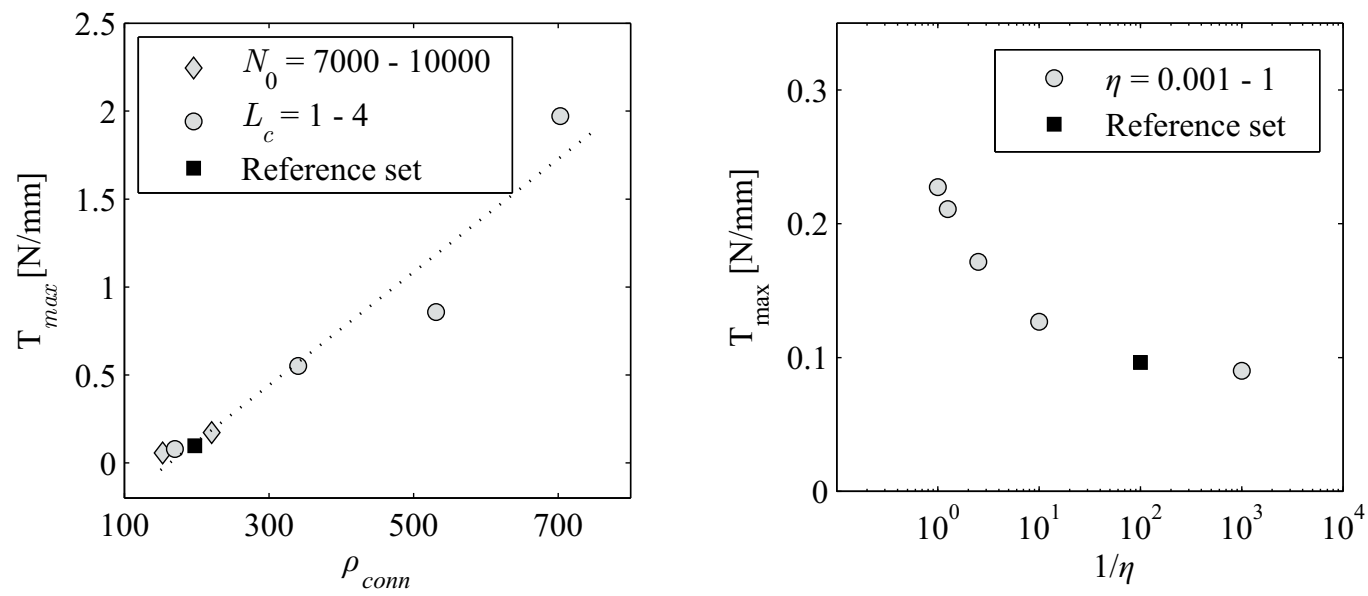

Figure 3: Sensitivity of the network response to geometric and damping parameters. The uniaxial tension $\mathrm{T}_{\max }$ computed at a global stretch $\lambda_{1}=1.3$ correlates to the connector density $\rho_{\text {conn }}$, for varying initial number of points $N_{0}$ and size of the discretization cells $L_{c}$, and to the numerical damping $\eta$. Reference set: $N_{0}=8000, L_{c}=1 \mathrm{~mm}$ and $\eta=$ $0.01 \mathrm{Ns} / \mathrm{mm}$. 


\subsection{Data extraction for comparison with experiments}

The network stretches in the imposed elongation direction, $\lambda_{1}$, and in the transverse direction, $\lambda_{2}$, are defined as $X / X_{0}$ or $Y / Y_{0}$, respectively, where $X$ and $Y$ represent the current length and width of the domain. The corresponding strains are defined as $\varepsilon=\lambda-1$. The connector stretch $\lambda_{\text {conn }}$ is computed as the ratio of the current to the initial connector length.

A scalar parameter $\mathrm{H}$ to quantify the degree of nonaffinity of the deformation is introduced, similarly to Hatami-Marbini and Picu (2008), as the average over all points of the ratio of $\left\|\mathbf{u}-\mathbf{u}^{a f f}\right\| /\left\|\mathbf{u}^{a f f}\right\|$, where $\mathbf{u}$ is the displacement at each point and $\mathbf{u}^{\text {aff }}$ is the corresponding displacement for a hypothetical affine deformation. In addition, the heterogeneity of the deformation field is visualized by the distribution of the area strain computed with a Delaunay triangulation algorithm. In particular, the Qhull (Qhull.org, 2015) implementation of the algorithm is used, which is available as a Python wrapper in the SciPy library (Jones et al., 2001). The DNM points, representing the crosslinks, are used to discretize the domain area in triangular elements with this algorithm. The change in area $\Delta A_{i}$ of each triangular element $i$ is calculated for each iteration step $n$ and is used to quantify the area strain $\varepsilon_{\text {areal }}^{n}=\Delta A_{i}^{n} / A_{0 i}$.

In order to facilitate the comparison with a corresponding analysis of MPM images, the orientation of the network microstructure is extracted with a image analysis method, similarly to Mauri et al. (2013). The distribution of orientation of the fibers is quantified with the OrientationJ plug-in for ImageJ (NIH, Bestheda, Maryland, USA) developed by Rezakhaniha et al. (2012). This tool extracts the distribution of the local angles $\alpha$ from gray- 
scale images and can be applied to the generated network and microscopy images alike. The distribution of connectors orientation is determined for selected loading states and reported as a probability distribution plot (Figs. 9 and 10).
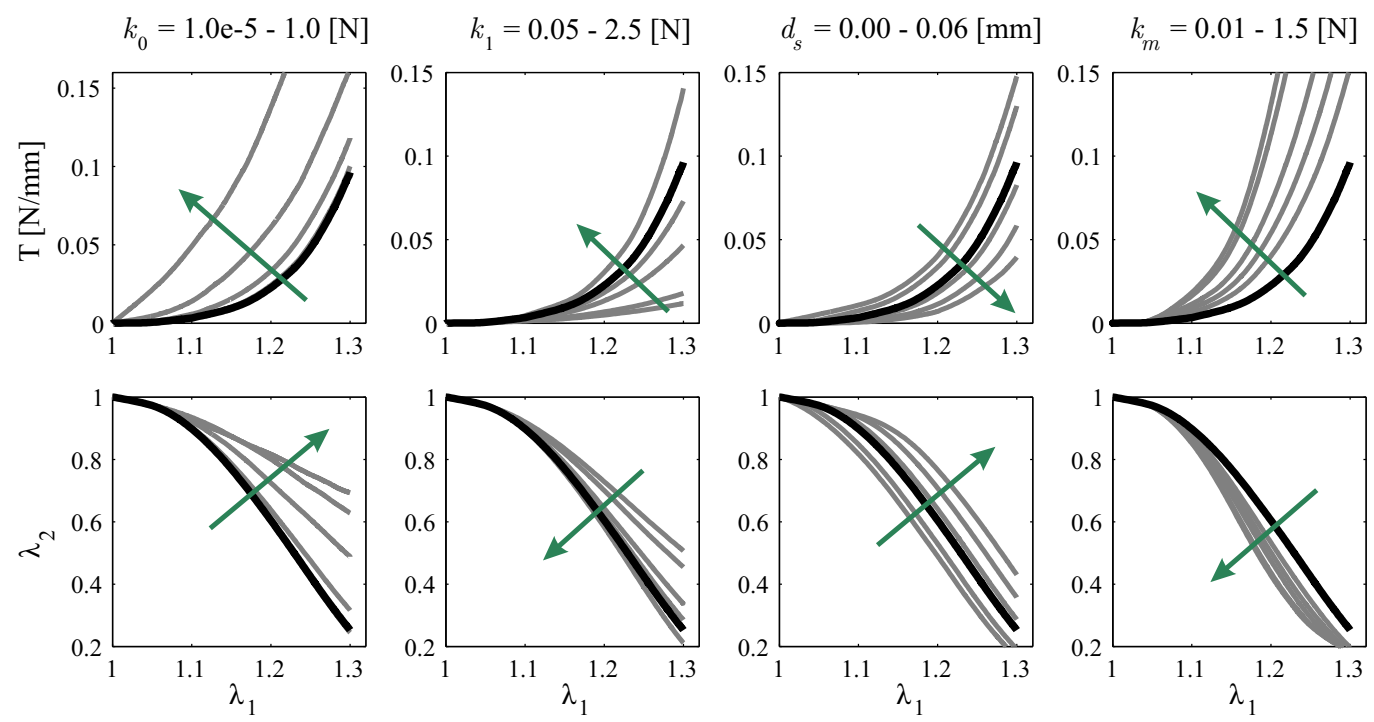

Figure 4: Sensitivity of the macroscopic response of the network to the behavior of the connectors in uniaxial tension (elongation: $\lambda_{1}$, transverse contraction: $\lambda_{2}$ ). Parameters are defined in Fig. 2e. The arrows indicate the shift of the curves for an increasing value of the varied parameter. The curves corresponding to the reference set are shown in black and the respective parameter set is reported in Table 1.

\section{Results}

\subsection{Parametric sensitivity study}

The effect of varying the model parameters on the predicted global response is analyzed first. 
Model size effect: The critical domain dimensions for which the results become independent of further increasing domain size has been determined by repeating the same simulation with increasing dimensions. The total strain energy of the domain normalized with respect to its size becomes size independent once $X_{0} / L_{c} \geq 10$. Note that the uniaxial and equibiaxial models used for the comparison with the experimental results have much larger domains with $X_{0} / L_{c}=80$ and $X_{0} / L_{c}=34$, respectively. Once the results converge, the total strain energy of the biaxial case is about one third of the strain energy of the same network forced to deform affinely; this observation has been reported repeatedly in the literature, e.g. Chandran and Barocas (2006).

Geometric parameters: Multiple networks generated through this stochastic process using the same input parameters differ only marginally in their macroscopic response. The sensitivity of the DNM to the geometrical input parameters is investigated next. The length of the discretization cells $L_{c}$ affects the tension-stretch response, which becomes stiffer with increasing $L_{c}$. This is due to the fact that the density of fibers correlates with the dimension of $L_{c}$. For the same initial number of points $N_{0}$, this implies higher connector density $\rho_{\text {conn }}$, which can be interpreted as higher collagen density. The dependency of the maximal nominal tension $\mathrm{T}_{\max }$ computed at a global stretch $\lambda_{1}=1.3$ on the connector density is shown in Fig. 3. Similarly, an increased $N_{0}$ at constant $L_{c}$ increases the connector density $\rho_{\text {conn }}$ and makes the behavior stiffer (see Fig. 3).

Connectors parameters: The effect of the connector parameters $k_{0}, k_{1}, d_{s}$ and $k_{m}$ is shown in Fig. 4. For each panel of Fig. 4 one parameter is var- 
ied while the others are kept at the reference value reported in Table 1. If the value of $k_{0}$ is enough small compared to $k_{1}$, the global response of the network becomes more non-linear and, as $k_{0}$ decreases, it converges to a strongly non-linear curve with high strain hardening at large strains. Also, large transverse contraction is observed for small values of $k_{0}$. The fiber stiffness under tension $\left(k_{1}\right)$ has a large effect on the large strain modulus of the macroscopic tension-stretch curve and a less strong effect on the transverse contraction. Interestingly, the stiffness of the fifth connector $\left(k_{m}\right)$ has a similar effect, but affects the tension-stretch curve only in the nonlinear transition regime. At higher stretches the softer fifth connector contributes little to the overall stiffness of the network, which is mainly given by the realigned stiffer fiber connectors. A larger value of $d_{s}$ results in a shift of the fiber recruitment process towards larger strains, leading to a long flat initial response phase and pronounced nonlinearity in the kinematics.

The macroscopic response resulting from a symmetric linear behavior of the connectors (i.e. $k_{0}=k_{1}=k_{m}$ ) is computed for higher and smaller stiffness values and compared to the reference set, see Fig. 5. The strong nonlinearity in both the tension-stretch and the kinematic responses is lost for such symmetrically linear characteristics independently of the stiffness value.

\subsection{Comparison to the response of human amnion}

The parameters reported in Table 1 and used as a reference for the parametric sensitivity study of the model has been selected to reproduce the uniaxial tension-stretch curve and the kinematic response of the amnion, reported in Bürzle and Mazza (2013). Note the excellent prediction of the equibiaxial response of the network (within the experimental variability) 

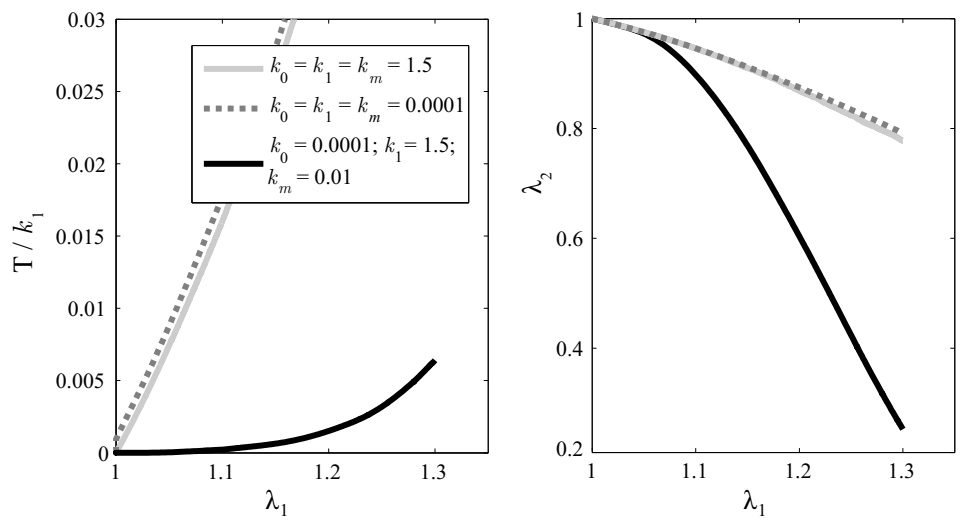

Figure 5: Nominal tension T normalized with the value of the parameter $k_{1}$ and kinematic response for linear and bilinear characteristics of the connectors. A more linear and stiffer response is visible for linear characteristics, independently of the $k_{1}$ value. Values in the figure legend are given in $\mathrm{N}$.

demonstrated in Fig. 6. In order to be consistent with the approach used to analyze the experimental data (cf. Bürzle and Mazza, 2013), a very low preforce is applied to the DNM to define the reference configuration.

Once this validation step is taken, we use the model to investigate physical quantities not accessible experimentally. The deformation is heterogeneous due to the discrete nature of the network. To show the level of heterogeneity we compute the local area strain between three adjacent points as explained in Sec. 2.4. The heterogeneity of the deformation field induced by the discrete nature of the network and by the nonlinear characteristics of the connectors is shown for both equibiaxial and uniaxial configurations at a global stretch of 1.15 (see Fig. 7a and 7b, respectively). The distribution of the connector stretch $\lambda_{\text {conn }}$ at the same global stretch is reported in Fig. 7c for each connector type. For uniaxial loading, many of the fifth connectors are loaded in compression $\left(\lambda_{\text {conn }}<1\right)$. This might result from the pronounced reduc- 
Figure 6: Representative network for the human amnion under uniaxial (UA) and equibiaxial (EB) loading conditions (model parameters cf. Table 1). The tension-stretch curves and the kinematic response fall within the standard deviation of the experimental curves (gray shadow) reported in Bürzle and Mazza (2013).

tion in area due to the large lateral contraction, compressing the linear fifth connector, which might be interpreted as a soft linear matrix element in the collagenous network. The contribution of the fifth connectors to the total deformation energy is small and the overall kinematics is controlled by the minimization of energy associated with the fiber connectors. Hence, system equilibrium may be reached with the linear fifth connector in unexpected deformation states. In the equibiaxial case, both connector types have a similar 
distribution with almost all connectors loaded in tension. The median value of $\lambda_{\text {conn }}$ for fiber connectors is 1.011 and 1.111 in uniaxial and equibiaxial cases, respectively. This indicates that the global stretch results mainly from rotation in uniaxial case, whereas in the equibiaxial case kinematics requires a more affine deformation.

The amount of nonaffinity in the equibiaxial case can be quantitatively assessed with the parameter $\mathrm{H}$, with $\mathrm{H}=0$ for affine deformation, and is shown in Fig. 8 for simulations of the model with linear and bilinear characteristics of the connectors. After a similar initial phase, the value of $\mathrm{H}$ increases considerably for the bilinear case $\left(k_{0}=0.0001 \mathrm{~N}, k_{1}=1.5 \mathrm{~N}\right.$ and $\left.k_{m}=0.01 \mathrm{~N}\right)$ compared to the linear one $\left(k_{0}=k_{1}=k_{m}=1.5 \mathrm{~N}\right)$. For $\lambda_{1}>1.05$ the bilinear connectors minimize their strain energy by rotating instead of stretching. This rearrangement increases the degree of nonaffinity of the deformation (i.e. increase in the parameter $\mathrm{H}$ ) and causes the strong lateral contraction visible in the global kinematic response of the uniaxial case (see Fig. 5). In fact, the kinematic response changes at the same deformation level, departing from the curve of the model with linear characteristic of the connectors at $\lambda_{1} \approx 1.05$. Note that rotation of connectors allow avoiding their stretch into the stiff range of their behavior, so that no pronounced stiffening is observed in the tension-stretch response.

The representative DNM shows strong fiber alignment during uniaxial elongation (see Fig. 9), which is significantly evident at $10 \%$ global strain and increases considerably for larger deformations. We note that realignment is minimal in the small-strain region of the network tension-strain curve (i.e. $\varepsilon_{1}<5 \%$ ). This finding is in agreement with the results of Fig. 8 , 
(a)

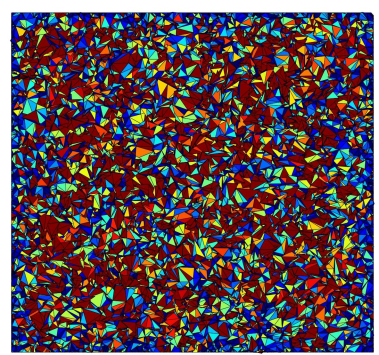

(b)

(c)
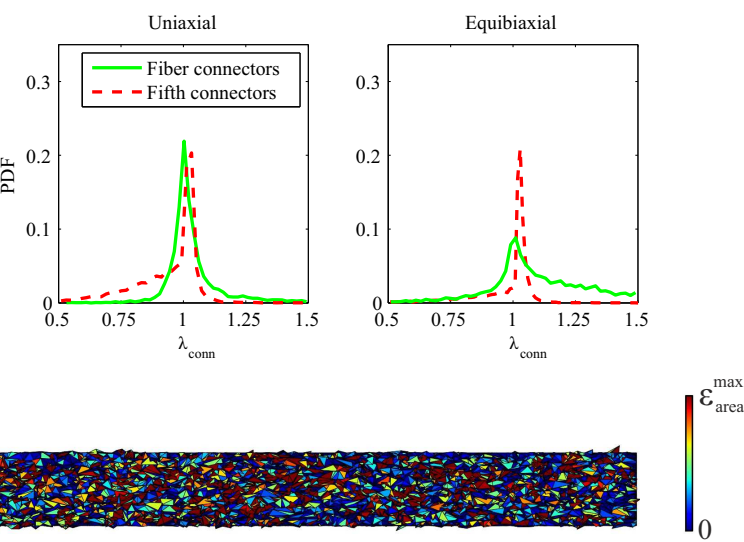

Figure 7: Extraction of the area strain from the Delaunay triangulation method to visualize the heterogeneity of the deformation field. The equibiaxial (a) and uniaxial (b) models are shown at a global stretch of 1.15 with the corresponding probability density function of the stretch in their connectors $\lambda_{\text {conn }}$ (c). The median of $\lambda_{\text {conn }}$ for fiber connectors in uniaxial and biaxial cases is 1.011 and 1.111 , respectively.

which detects the nonaffinity associated with network rearrangement only for $\varepsilon_{1}>5 \%$. Hence, the geometrically induced nonlinearity dominates the network behavior beyond a critical strain, as has been observed repeatedly in the literature (e.g. Chandran and Barocas, 2006). Moreover, the observation of progressive fiber alignment are in good agreement with the collagen reorientation observed in situ by MPM (Mauri et al., 2013, 2015a).

\subsection{Local deformation at a circular defect}

The effect of a circular hole in the center of a large DNM domain (approx. $45^{\prime} 000$ points and 116'000 connectors) under equibiaxial load is shown for the entire domain (Fig. 10a) and for a close up view of the region oriented perpendicular to the boundary of the hole (Fig. 10b) at an equibiaxial global stretch of $\lambda_{1}=1.3$. We remind that the connectors within the hole are 
Figure 8: Nonaffinity parameter $\mathrm{H}$ over the global elongation strain $\lambda_{1}$ for the equibiaxial configuration. After the initial phase, considerably higher nonaffinity is visible for bilinear rather than linear connector characteristics. Values in the legend are given in N.

still present to ensure physical stability of the network, but their stiffness is reduced by two orders of magnitude so that their contribution is negligible. The orientation at the border of the hole is analyzed within different areas (Fig. 10b) and demonstrates strong alignment of the fibers in the direction tangential to the circular defect only in a very small area near the hole boundary $(\mathrm{NF})$, while alignment is absent in a proximal region (MF) and in the far field $(\mathrm{FF})$. The change in diameter of the hole was used to evaluate the circumferential stretch at the edge, which reached values of 1.31 and 1.74 for $\lambda_{1}=1.11$ and $\lambda_{1}=1.3$, respectively. 

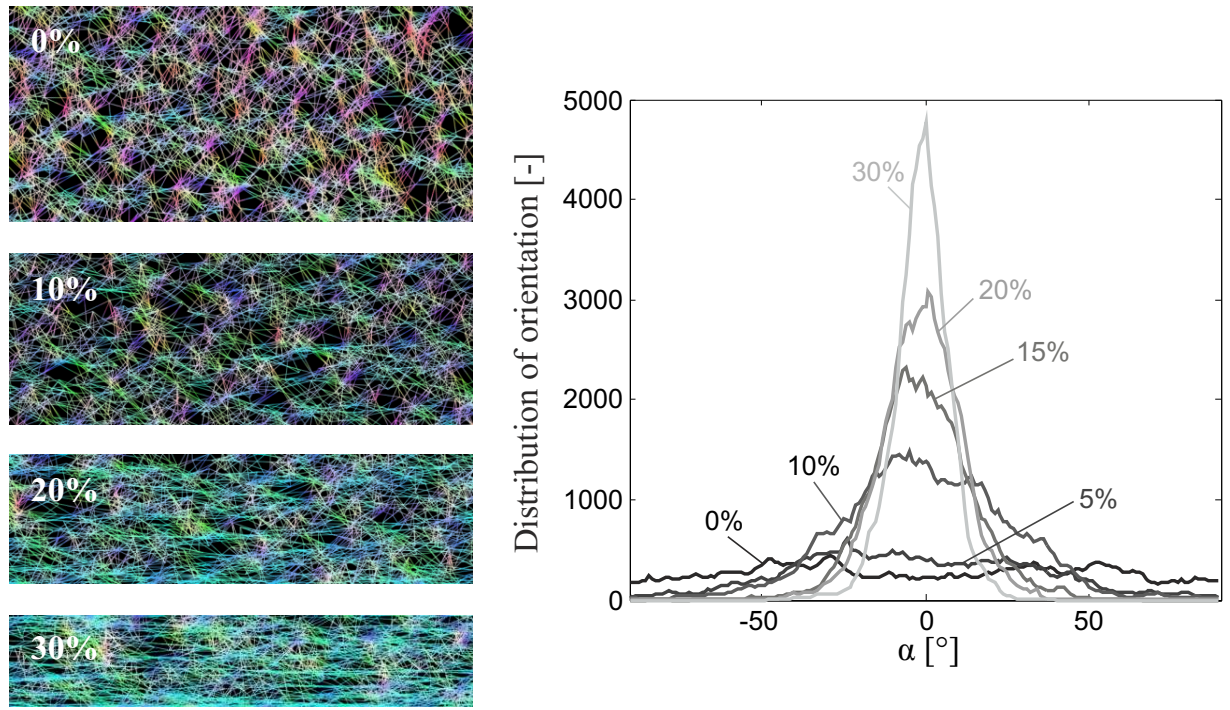

Figure 9: Orientation analysis of the central region of the DNM under uniaxial elongation. Orientation angles of the connectors are extracted directly from the images (with ImageJ, see Sec. 2.4) at different values of global strain. The colors of the images represents the orientation of the identified structure with the image analysis algorithm (cyan is for $\alpha=0^{\circ}$ and represents the global elongation direction, whereas red is for $\alpha=90^{\circ}$, i.e. the transverse direction). The network microstructure aligns significantly around the direction of elongation $\left(\alpha=0^{\circ}\right)$ already at $10 \%$ global strain.

\section{Discussion}

\subsection{Representation of the mechanical response of the amnion}

The mechanical behavior of the human amnion can be described by the present DNM composed of points and connectors, which represent the crosslinked collagenous structure of this tissue. The macroscopic response of the proposed DNM is in good agreement with experimental data from Bürzle and Mazza (2013), see Fig. 6. Moreover, deformation mechanisms activated in uniaxial tension are in line with in situ experimental results based on MPM 
(Mauri et al., 2013, 2015a). The large and progressive collagen alignment, especially at low to modest deformations, is reproduced by the model (see Fig. 9) and represents its key toughening mechanism (cf. Koh et al., 2013) at the edge of a hole. The large annular compaction of the network at the border of the hole (see Fig. 10) enlarges the defect, resulting in a circumferential stretch of about 1.3 for a global stretch of 1.11. These values are in line with experimental observations from inflated fetal membranes with a circular defect in the amnion (W. Bürzle, unpublished data).

The model parameters were determined based on data from uniaxial tension tests and the much stiffer equibiaxial response of amnion observed in the experiments was successfully predicted. The response of the model falls within the standard deviation of the experiments demonstrating that it is possible to include the main deformation mechanisms in this simple and efficient discrete model. The extraordinary large transverse contraction characteristics of amnion (Bürzle and Mazza, 2013) was associated in the model with a strong realignment of the connectors towards the direction of elongation (see Fig. 9). The overestimation of the transverse contraction at very high deformations might be due to the fact that no volume is attributed to the fibers, whereas in the experiments further compaction of the collagen network might be restrained by the space occupied by the fibers (Mauri et al., 2015b,a). As proposed in Bürzle and Mazza (2013) for a phenomenological model of the amnion, the high degree of reorientation of fibers is related to their low resistance to compressive forces, i.e. buckling. This is confirmed by the results in Fig. 4, showing reduced transverse contraction when $k_{0}$ and $k_{1}$ become comparable, as well as for large $d_{s}$. Similarly, Fig. 5 demonstrates 
that nonlinearity of the tension-stretch curve and large transverse contraction are due to the higher compression compliance relative to the compliance in tension of fiber connectors. Further, the parameters $k_{1}$ and $k_{0}$ are shown to determine the high and low strain response, while the transition depends more significantly on the parameters $k_{m}$ and $d_{s}$.

In uniaxial tension experiments, amnion is characterized by a long initial phase, in which the forces are extremely low and hardly measurable whereas kinematic mechanisms are clearly activated, adapting the tissue to the new loading state (cf. e.g. Mauri et al., 2015a). The experimental curves are therefore referred to the configuration at a small preforce level (cf. Bürzle and Mazza, 2013) to reduce the scatter and exclude this initial region. The DNM presented in here is able to capture this transition, characterized by an adaptive reorientation of the network at very low strain energy, which also results in the initial nonlinearity of the kinematics (see Figs. 4 and 5).

While the macroscopic deformation behavior of amnion can adequately be represented with a phenomenological continuum models (Bürzle and Mazza, 2013), the DNM provides the possibility to investigate local strain heterogeneity and nonaffine deformations, especially important for the analysis of defects (Koh and Oyen, 2015). The proposed DNM is not directly based on the observed microstructure, i.e. on microscopy images, as e.g. D'Amore et al. (2014), but rather on a phenomenological representation of a discrete network with physically motivated parameters and connector properties that can be related to experimental observations. In fact, the stiffer response of the network for larger $L_{c}$, i.e. for increased collagenous fiber density (see Fig. 3), is in line with the positive correlation between the amount of colla- 
gen and the stiffness of the macroscopic tension-stretch response reported in Jabareen et al. (2009) and Bürzle et al. (2013). Similarly, the positive correlation between the crosslinks density and the high stretch stiffness of the amnion (Bürzle et al., 2013) is confirmed by the network model (for varying $N_{0}$ ), and is in accordance with previous studies (Sopakayang et al., 2012; Puxkandl et al., 2002; Hansen et al., 2009). However, we remark that $N_{0}$ is a parameter of the representative network and its numeric value does not directly compare to estimates of collagen cross-linkage obtained in biochemical analyses. Moreover, the shift of the recruitment stretch of the fiber associated with the parameter $d_{s}$ might be used to represent the progressive fiber undulation due to repeated loading during e.g. early contractions (cf. Mauri et al., 2013).

\subsection{Stiffening effect in the macroscopic response}

The nonlinear stiffening is an intrinsic property of structural networks, able to activate the most convenient deformation regime, minimizing the energy-expensive stretching of single filaments. This deformation regime might differ, depending on the microstructure of the network and on the modeling assumption made for representing the fibers: bending vs. stretching for rigid joints (e.g Onck et al., 2005; Huisman et al., 2007; Kurniawan et al., 2012), rotation and bending vs. stretching for pin-joints (e.g Chandran and Barocas, 2006), or stretching of the compliant crosslinks (represented with a soft multiaxial spring) vs. bending and stretching of the fibers (e.g Zagar et al., 2015). In our DNM, the bending regime under compressive and low tensile forces is considered in the axial connectors by the parameter $k_{0}$, allowing the network to switch between bending- and tension-dominated 
(a)

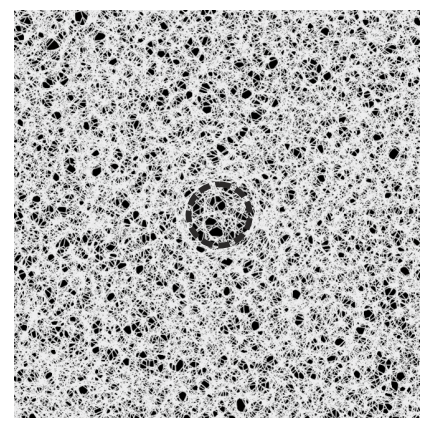

(b)

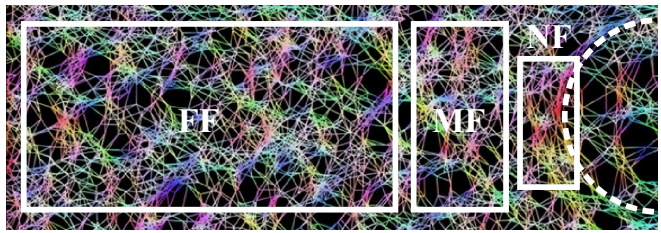

(c)

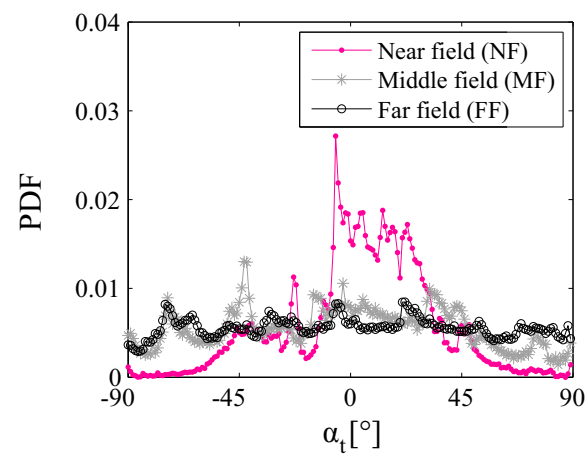

Figure 10: Response of the entire DNM (a) with a hole (highlighted with a dashed line) under global equibiaxial stretch $\left(\lambda_{1}=1.3\right)$ and a close up view (b) of the region perpendicular to the hole (b, dashed line). The connectors in the hole have a stiffness two orders of magnitude smaller than the rest of the network. The orientation of the network microstructure in the far field (FF), middle field (MF) and near field (NF) is extracted directly from the image (with ImageJ, see Sec. 2.4) and is represented in its colors. Red is for $\alpha_{t}=0^{\circ}$ and represents the direction tangential to the hole edge, whereas cyan is for $\alpha_{t}=90^{\circ}$ (i.e. perpendicular to the hole edge). The corresponding probability distribution functions (c) show a pronounced alignment of the connectors tangential to the hole edge confined in a very small region (NF).

modes and capturing this nonlinear stiffening. In the DNM presented in this paper, the fiber is assumed to consist of a linear-elastic material (stiffness $\left.k_{1}\right)$ taking a wavy form in the networks reference state. Bending is assumed to govern the compressive and straightening response of the fibers, lumped into an effective axial stiffness that is represented by the parameter $k_{0}$ of the axial connector elements. This bi-linear representation of axial fiber force allows the network to switch between bending- and tension-dominated modes 
and to capture the nonlinear stiffening. The stabilization of the macroscopic response for small values of $k_{0}$ (i.e. the response does not change for even smaller values of $k_{0}$ ) suggests that for sufficiently large differences between bending and tensile stiffness, the network is able to rearrange its microstructure at very low deformation energy. On the other hand, simulations with the same stiffness in tension and bending regimes resulted in a nearly linear macroscopic response of the network (see Fig. 5). Thus, the nonlinear stiffening effect strongly relates to the behavior of the network filaments and is less sensitive to the geometrical constraints of the network, which are representative of e.g. collagen concentration (Licup et al., 2015). The fact that most biological tissues under physiological conditions are loaded in the nonlinear regimen of the stress-stretch curve might indicate that the nonlinearity of this transition is characteristic of the tissue functionality (Pritchard et al., 2014) and strongly depends on the structural properties of its microstructure, given by the organization of the collagen fibrils in the fibers and their waviness.

\subsection{Microstructural reorientation in uniaxial loading}

The rearrangement of the connectors can be quantified similarly to the reorientation of collagen fibers observed in situ in by MPM (Mauri et al., 2015a,b; Perrini et al., 2015). The progression of fiber alignment seen in amnion is reflected in the DNM by a narrowing distribution of the orientation angle around the direction of loading in uniaxial tension (see Fig. 10). This effect is indicative since the stress state in the near field of a defect (e.g. a hole or a crack after blunting) is uniaxial and fibers will strongly align in this region. The visible adaptation of the microstructure at the boundary of a 
circular hole (see Fig. 10) increases the mechanical support in the direction of maximal loading and is consistent with the experimental observations by Koh et al. (2013) for tough membranes. The microstructure is visibly oriented in tangential direction and compacted to higher density at the hole, resulting in a circumferential stretch of about 1.7 at the global stretch of $\lambda_{1}=1.3$. Note that already at a relatively small distance from the hole boundary (MF in Fig. 10, at a distance less that the hole radius) no realignment is present, indicating that the compaction mechanisms confines the notch near field (NF in Fig. 10) to a very small region at the border of the hole.

\subsection{Limitations of the model}

The choice of a planar network was motivated by the microstructure of the amnion, for which most fibers lie in the plane (no fibers run in throughthickness direction) and create a multilayered structure with a thickness of less than $150 \mu \mathrm{m}$ (Fawthrop and Ockleford, 1994; Jabareen et al., 2009; Mauri et al., 2015a). For this reason, a three-dimensional extension of the DNM might not be required for this material.

The proposed model was not designed to include the time-dependent behavior of the amnion (Lavery and Miller, 1977; Oyen et al., 2004; Mauri et al., 2015b). However, the damping component of the connectors could be calibrated to address the experimentally observed time- and history-dependence of the amnion (Mauri et al., 2015b; Perrini et al., 2015). In particular, viscoelastic behavior was associated with water movement and fiber dissipation (Mauri et al., 2015b) and these effects may be captured by introducing damping in the constitutive equation of fibers. Similar to the approach of Koh and Oyen (2015), damage processes such as the progressive failure of the collage- 
nous fibers, could be implemented in this framework through the connector behavior, e.g. by defining critical stress or strain values. In this case, the physical stability of the network should be verified and ensured even after the progressive failure of fibers. This type of DNM would allow the quantitative investigation of the correlation between critical tension, collagen density and crosslink density measured for the amnion (Bürzle et al., 2013). The proposed DNM represents the collagen fibers phenomenologically by connector elements. This approach models the bending mode during fiber uncrimping and the tension mode after fiber straightening both leading to an effective axial force, but neglects the potential bending of straight fibers. Representing the geometry and mechanical properties of the fibers in more detail would enable computing the mechanical response of the network based on tensile and bending properties of the single collagen fibers from experimental investigations (e.g. Hansen et al., 2009).

\section{Conclusion}

The proposed DNM is an efficient model to represent the mechanical behavior of the human amnion. The tension-stretch nonlinearity and the inplane kinematics of the macroscopic experiments are reproduced for uniaxial and biaxial loading conditions. The fiber network deformation mechanisms are in line with observations from in situ mechanical tests with MPM as well as macroscopic experiments. The model is applied to the example of a circular defect, a configuration which might be relevant in clinical situations such as fetoscopic interventions or amniocentesis. Future studies might directly compare simulations based on this DNM with corresponding in situ experi- 
ments, focusing on the local strain field at the hole or on the effect of defect geometries. The developed framework is a useful and versatile tool, which can be applied to other fibrous biomembranes to investigate their mechanical behavior at different length scales.

\section{Acknowledgment}

The authors are grateful to the Swiss National Science Foundation (SNSF) for financial support (Project number: 205321 134803/1). AEE gratefully acknowledges the support within the ETH Zurich Postdoctoral Fellowship (FEL13-12-2) and Marie Curie Actions for People COFUND programs. We thank K. Bircher (ETH Zurich) for his support in cross-checking the numerical computations.

\section{References}

Abhilash, A., Baker, B. M., Trappmann, B., Chen, C. S., Shenoy, V. B., 2014. Remodeling of fibrous extracellular matrices by contractile cells: Predictions from discrete fiber network simulations. Biophysical Journal 107 (8), 1829-1840.

URL http://www. sciencedirect.com/science/article/pii/S000634951400931X

Broedersz, C. P., MacKintosh, F. C., 2014. Modeling semiflexible polymer networks. Reviews of Modern Physics 86 (3), 995-1036.

Bürzle, W., Haller, C. M., Jabareen, M., Egger, J., Mallik, A. S., OchsenbeinKölble, N., Ehrbar, M., Mazza, E., 2013. Multiaxial mechanical behavior 
of human fetal membranes and its relationship to microstructure. Biomechanics and Modeling in Mechanobiology 12, 747-762.

URL http://dx.doi.org/10.1007/s10237-012-0438-z

Bürzle, W., Mazza, E., 2013. On the deformation behavior of human amnion. Journal of Biomechanics 46 (11), 1777-1783.

URL http://www.sciencedirect.com/science/article/pii/S0021929013002492

Carleton, J. B., D’Amore, A., Feaver, K. R., Rodin, G. J., Sacks, M. S., 2015.

Geometric characterization and simulation of planar layered elastomeric fibrous biomaterials. Acta Biomaterialia 12, 93-101.

URL http://www.sciencedirect.com/science/article/pii/S1742706114004413

Chandran, P. L., Barocas, V. H., 2006. Affine versus non-affine fibril kinematics in collagen networks: Theoretical studies of network behavior. Journal of Biomechanical Engineering 128 (2), 259-270.

URL http://dx.doi.org/10.1115/1.2165699

Chandran, P. L., Barocas, V. H., Aug. 2007. Deterministic material-based averaging theory model of collagen gel micromechanics. Journal of Biomechanical Engineering 129 (2), 137-147.

URL http://dx.doi.org/10.1115/1.2472369

D'Amore, A., Amoroso, N., Gottardi, R., Hobson, C., Carruthers, C., Watkins, S., Wagner, W. R., Sacks, M. S., 2014. From single fiber to macro-level mechanics: A structural finite-element model for elastomeric fibrous biomaterials. Journal of the Mechanical Behavior of Biomedical 
Materials 39, 146-161.

URL http://www.sciencedirect.com/science/article/pii/S1751616114002094

Fawthrop, R. K., Ockleford, C. D., 1994. Cryofracture of human term amniochorion. Cell and Tissue Research 277 (2), 315-323.

URL http://dx.doi.org/10.1007/BF00327779

Hadi, M. F., Barocas, V. H., 2013. Microscale fiber network alignment affects macroscale failure behavior in simulated collagen tissue analogs. Journal of biomechanical engineering 135 (2), 021026.

Hansen, P., Hassenkam, T., Svensson, R. B., Aagaard, P., Trappe, T., Haraldsson, B. T., Kjaer, M., Magnusson, P., 2009. Glutaraldehyde crosslinking of tendon-mechanical effects at the level of the tendon fascicle and fibril. Connective tissue research 50 (4), 211-222.

Hatami-Marbini, H., Picu, R. C., 2008. Scaling of nonaffine deformation in random semiflexible fiber networks. Physical Review E 77 (6), 062103.

Hollenstein, M., 2011. Mechanics of the human liver: Experiments and modeling. Ph.D. thesis, Eidgenössische Technische Hochschule ETH Zürich, Diss. Nr. 19587.

Huisman, E., Van Dillen, T., Onck, P., Van der Giessen, E., 2007. Threedimensional cross-linked f-actin networks: relation between network architecture and mechanical behavior. Physical review letters 99 (20), 208103.

Isaksson, P., Hägglund, R., 2009. Structural effects on deformation and fracture of random fiber networks and consequences on continuum models. 
International Journal of Solids and Structures 46 (11-12), 2320-2329.

URL http://www.sciencedirect.com/science/article/pii/S0020768309000432

Jabareen, M., Mallik, A. S., Bilic, G., Zisch, A. H., Mazza, E., 2009. Relation between mechanical properties and microstructure of human fetal membranes: An attempt towards a quantitative analysis. European Journal of Obstetrics \& Gynecology and Reproductive Biology 144 (Suppl.1), S134-S141.

URL http://www.sciencedirect.com/science/article/pii/S030121150900147X

Jones, E., Oliphant, T., Peterson, P., et al., 2001. SciPy: Open source scientific tools for Python. [Online; accessed 2015-05-18].

URL http: //www.scipy.org/

Koh, C., Oyen, M., 2012a. Fracture toughness of fibrous membranes. Technische Mechanik 32 (2-5), 333-341.

Koh, C., Strange, D., Tonsomboon, K., Oyen, M., 2013. Failure mechanisms in fibrous scaffolds. Acta Biomaterialia 9 (7), 7326-7334.

URL http://www.sciencedirect.com/science/article/pii/S1742706113001165

Koh, C. T., Oyen, M. L., 2012b. Branching toughens fibrous networks. Journal of the Mechanical Behavior of Biomedical Materials 12, 74-82. URL http://www.sciencedirect.com/science/article/pii/S1751616112000914

Koh, C. T., Oyen, M. L., 2015. Toughening in electrospun fibrous scaffolds. APL Materials 3 (1), 014908. URL http://scitation.aip.org/content/aip/journal/aplmater/3/1/10.1063/1.4901450 
Kulachenko, A., Uesaka, T., 2012. Direct simulations of fiber network deformation and failure. Mechanics of Materials 51, 1-14.

URL http://www.sciencedirect.com/science/article/pii/S0167663612000683

Kurniawan, N. A., Enemark, S., Rajagopalan, R., 2012. The role of structure in the nonlinear mechanics of cross-linked semiflexible polymer networks. The Journal of chemical physics 136 (6), 065101.

Lake, S. P., Barocas, V. H., 2011. Mechanical and structural contribution of non-fibrillar matrix in uniaxial tension: A collagen-agarose co-gel model. Annals of Biomedical Engineering 39 (7), 1891-1903.

URL http://dx.doi.org/10.1007/s10439-011-0298-1

Lavery, J. P., Miller, C. E., 1977. The viscoelastic nature of chorioamniotic membranes. Obstetrics and Gynecology 50 (4), 467-472.

Licup, A. J., Münster, S., Sharma, A., Sheinman, M., Jawerth, L. M., Fabry, B., Weitz, D. A., MacKintosh, F. C., 2015. Stress controls the mechanics of collagen networks. arXiv preprint arXiv:1503.00924 -, in press.

Mauri, A., Ehret, A. E., Perrini, M., Maake, C., Ochsenbein-Kölble, N., Ehrbar, M., Oyen, M. L., Mazza, E., 2015a. Deformation mechanisms of human amnion: Quantitative studies based on second harmonic generation microscopy. Journal of Biomechanics (0), -.

URL http://www.sciencedirect.com/science/article/pii/S0021929015000615

Mauri, A., Perrini, M., Ehret, A. E., Focatiis, D. S. D., Mazza, E., 2015b.

Time-dependent mechanical behavior of human amnion: Macroscopic and 
microscopic characterization. Acta Biomaterialia 11, 314-323.

URL http://www.sciencedirect.com/science/article/pii/S174270611400395X

Mauri, A., Perrini, M., Mateos, J., Maake, C., Ochsenbein-Kölble, N., Zimmermann, R., Ehrbar, M., Mazza, E., 2013. Second harmonic generation microscopy of fetal membranes under deformation: Normal and altered morphology. Placenta 34 (11), 1020-1026.

URL http://www.sciencedirect.com/science/article/pii/S0143400413007285

Maxwell, J. C., 1864. On the calculation of the equilibrium and stiffness of frames. The London, Edinburgh, and Dublin Philosophical Magazine and Journal of Science 27 (182), 294-299.

Onck, P. R., Koeman, T., Van Dillen, T., Van der Giessen, E., 2005. Alternative explanation of stiffening in cross-linked semiflexible networks. Physical review letters 95 (17), 178102.

Oyen, M. L., Calvin, S. E., Cook, R. F., 2004. Uniaxial stress-relaxation and stress-strain responses of human amnion. Journal of Materials Science: Materials in Medicine 15 (5), 619-624.

Oyen, M. L., Cook, R. F., Stylianopoulos, T., Barocas, V. H., Calvin, S. E., Landers, D. V., 2005. Uniaxial and biaxial mechanical behavior of human amnion. Journal of Materials Research 20 (11), 2902-2909.

URL http://dx.doi.org/10.1557/JMR.2005.0382

Perrini, M., Mauri, A., Ehret, A. E., Ochsenbein-Kölble, N., Zimmermann, R., Ehrbar, M., Mazza, E., 2015. Mechanical and microstructural investigation of the cyclic behavior of human amnion. Journal of Biomechanical 
Engineering 137 (6), 061010.

URL http://dx.doi.org/10.1115/1.4030054

Picu, R. C., 2011. Mechanics of random fiber networks-a review. Soft Matter 7 (15), 6768-6785.

URL http://dx.doi.org/10.1039/C1SM05022B

Pritchard, R. H., Huang, Y. Y. S., Terentjev, E. M., 2014. Mechanics of biological networks: from the cell cytoskeleton to connective tissue. Soft matter 10 (12), 1864-1884.

Puxkandl, R., Zizak, I., Paris, O., Keckes, J., Tesch, W., Bernstorff, S., Purslow, P., Fratzl, P., 2002. Viscoelastic properties of collagen: synchrotron radiation investigations and structural model. Philosophical Transactions of the Royal Society of London. Series B: Biological Sciences 357 (1418), 191-197.

Qhull.org, 2015. Qhull code for convex hull, delaunay triangulation, voronoi diagram, and halfspace intersection about a point.

URL http://www.qhull.org

Rezakhaniha, R., Agianniotis, A., Schrauwen, J. T. C., Griffa, A., Sage, D., Bouten, C. V. C., van de Vosse, F. N., Unser, M., Stergiopulos, N., 2012. Experimental investigation of collagen waviness and orientation in the arterial adventitia using confocal laser scanning microscopy. Biomechanics and Modeling in Mechanobiology 11 (3-4), 461-473.

URL http://dx.doi .org/10.1007/s10237-011-0325-z 
Shah, S. B., Witzenburg, C., Hadi, M. F., Wagner, H. P., Goodrich, J. M., Alford, P. W., Barocas, V. H., 2014. Prefailure and failure mechanics of the porcine ascending thoracic aorta: experiments and a multiscale model. Journal of biomechanical engineering 136 (2), 021028.

Shahsavari, A., Picu, R., 2013. Size effect on mechanical behavior of random fiber networks. International Journal of Solids and Structures 50 (20-21), $3332-3338$.

URL http://www.sciencedirect.com/science/article/pii/S0020768313002382

Shahsavari, A., Picu, R. C., 2012. Model selection for athermal cross-linked fiber networks. Physical Review E 86, 011923.

URL http://link.aps.org/doi/10.1103/PhysRevE.86.011923

Sopakayang, R., De Vita, R., Kwansa, A., Freeman, J. W., 2012. Elastic and viscoelastic properties of a type i collagen fiber. Journal of Theoretical Biology 293, 197-205.

URL http://www.sciencedirect.com/science/article/pii/S0022519311005339

Stylianopoulos, T., Barocas, V. H., 2007a. Multiscale, structure-based modeling for the elastic mechanical behavior of arterial walls. Journal of biomechanical engineering 129 (4), 611-618.

Stylianopoulos, T., Barocas, V. H., 2007b. Volume-averaging theory for the study of the mechanics of collagen networks. Computer Methods in Applied Mechanics and Engineering 196 (31-32), 2981-2990.

URL http://www.sciencedirect.com/science/article/pii/S0045782507000333 
Wang, H., Abhilash, A., Chen, C. S., Wells, R. G., Shenoy, V. B., 2014. Long-range force transmission in fibrous matrices enabled by tensiondriven alignment of fibers. Biophysical Journal 107 (11), 2592-2603.

URL http://www.sciencedirect.com/science/article/pii/S0006349514011096

Zagar, G., Onck, P. R., van der Giessen, E., 2015. Two fundamental mechanisms govern the stiffening of cross-linked networks. Biophysical Journal 108 (6), 1470-1479.

URL http://www.sciencedirect.com/science/article/pii/S0006349515001782

Zhang, L., Lake, S. P., Barocas, V. H., Shephard, M. S., Picu, R. C., 2013a. Cross-linked fiber network embedded in an elastic matrix. Soft matter 9 (28), 6398-6405.

Zhang, L., Lake, S. P., Lai, V. K., Picu, C. R., Barocas, V. H., Shephard, M. S., 2013b. A coupled fiber-matrix model demonstrates highly inhomogeneous microstructural interactions in soft tissues under tensile load. Journal of biomechanical engineering 135 (1), 011008. 


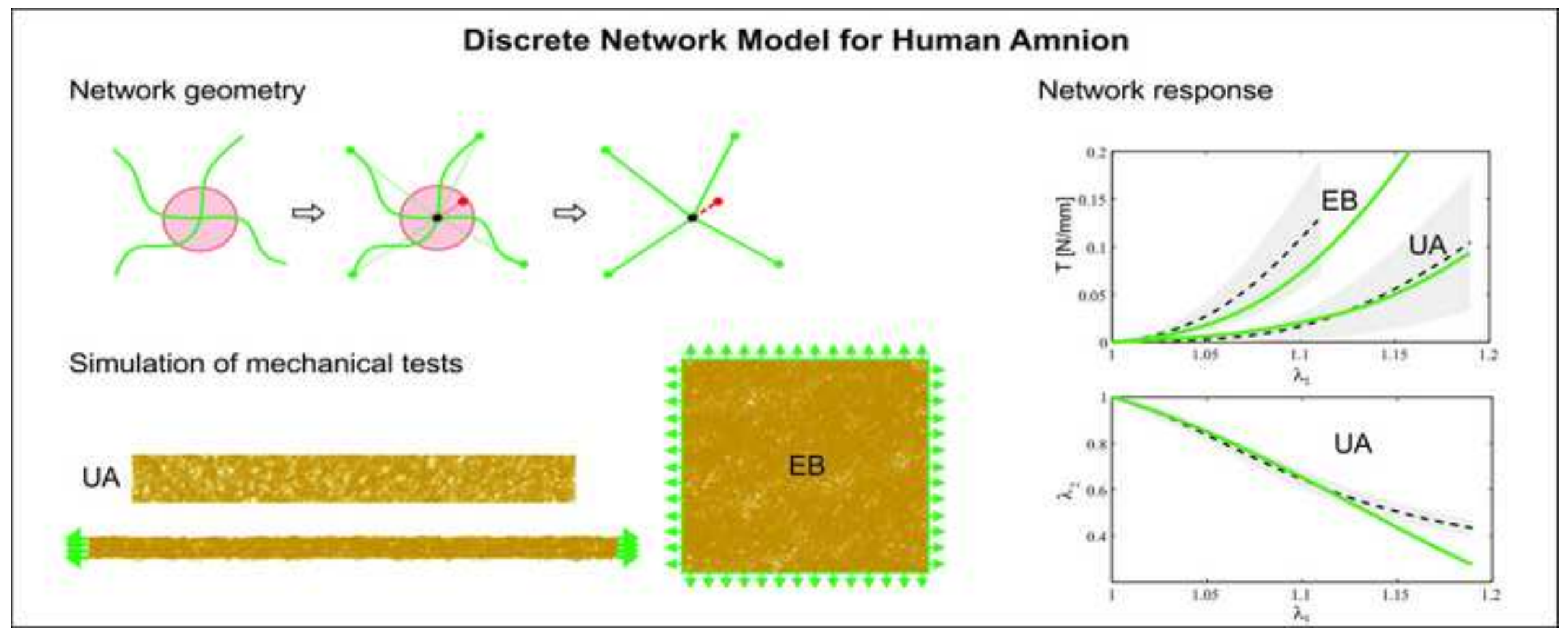

Simulation of mechanical tests

UA 